\title{
Über die chemische Zusammensetzung des Dysanalyts von Vogtsburg im Kaiserstuhl.
}

\author{
Von \\ W. Meigen und E. Huger.
}

Der Dysanalyt von Vogtsburg im Kaiserstuhl ist lange Zeit für Perowskit gehalten worden, bis KNOP ${ }^{1}$ darin einen gröBeren Gehalt an Niobsäure, sowie an seltenen Erden fand und ihm daher den neuen Namen Dysanalyt gab, wegen der besonderen Schwierigkeiten der Analyse.

Nach Krop ist der Dysanalyt im wesentlichen eine isomorphe Mischung von Metatitanaten mit Niobaten, wobei die Base hauptsächlich Kalk, außerdem Eisenoxydul, Ceroxyd und Natron ist. Der Dysanalyt würde hiernach eine Mittelstellung einnehmen zwischen dem Perowskit, dem reinen Titanat einerseits, und dem gleichfalls im Kaiserstuhl vorkommenden Koppit, dem Niobat andererseits. Der Koppit enthält auch noch Fluornatrium und schlieBt sich dadurch an die Pyrochlormineralien an.

In jüngster Zeit ist der Dysanalyt von Vogtsburg von HAUSER ${ }^{2}$ analysiert worden. Die von HAUSER gefundenen Zahlen weichen von denen der KNopschen Analyse ziemlich bedeutend ab. Krop fand die unter 1, HaUSER die unter 2 angegebenen Zahlen:

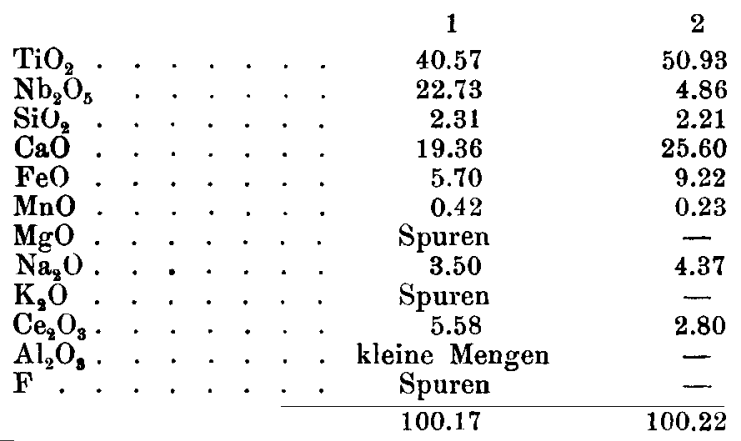

1 Krop, Dysanalyt, ein pyrochlorartiges Mineral. Zeitschr. f. Krist. 1 (1877), 3.

2 O. HAUser, Über den sogen. Dysanalyt von Vogtsburg im Kaiserstuhl. Z. anorg. Chem. 60 (1908), 237. 
Den Unterschied im Niobgehalt glaubt HaUser auf die von KNop angewandte mangelhafte Trennungsmethode für Niob und Titan zurückführen zu müssen. Bei den übrigen Bestandteilen nimmt er an, daB das Mineral nicht einheitlich sei und daher eine wechselnde Zusammensetzung besitze. Auf Grund seiner Analyse kommt HaUSER zu dem SchluB, daB der Dysanalyt keine selbständige Mineralart ist, sondern ein durch Einschlüsse verunreinigter Perowskit, dessen Gehalt an Niobsäure und Ceriterden nur auf dem Vorhandensein eben dieser Einschlüsse beruhe.

Die von HaJSER angewandte Trennungsmethode ist auch nicht einwandsfrei. Nach unseren Versuchen erhält man danach einen viel zu niedrigen Niobgehalt. Es schien uns daher eine erneute Analyse des Dysanalyts nicht überfiüssig, zumal wir durch die Güte von Herrn Prof. Dr. G. Mexer in Freiburg i. Br. über ein ungewöhnlich reiches Material verfügen konnten, für das wir ihm auch an dieser Stelle unseren besten Dank aussprechen. Sowohl KNop wie HAUSER hatten nur eine verhältnismäBig geringe Menge Material für ihre Untersuchungen zur Verfügung, KNoP gegen $50 \mathrm{~g}$, HAUSER etwa $20 \mathrm{~g}$, das außerdem offenbar auch nicht ganz rein gewesen ist. Herr Prof. MeYer hat durch Auflösen von etwa $800 \mathrm{~kg}$ des körnigen Kalksteins von Vogtsburg im ganzen etwa $4 \mathrm{~kg}$ rohen Dysanalyt gewonnen. Für die Analyse konnten so nur die allerreinsten und besten Kristalle ausgelesen werden.

Das uns zur Verfügung stehende Material war bereits in weitgehender Weise durch elektromagnetische Aufbereitung und Auslesen gereinigt. Die noch verunreinigenden Bestandteile waren Quarz, Pseudobiotit, Brauneisenerz und Pyroxen. Das Gemenge wurde durch Kochen mit verdünnter Salzsäure und nachherige Behandlung mit Kalilauge noch weiter gereinigt. Kristallisierter Dysanalyt wird von diesen Reagenzien nicht angegriffen; wenn er sehr fein gepulvert wird, löst ihn konzentrierte Salzsäure teilweise auf. Aus dem verbleibenden Rückstande wurden die Dysanalytkristalle mit der Pinzette herausgelesen und unter der Lupe die besten davon ausgesucht.

Der Dysanalyt kristallisiert stets in gut ausgebildeten Würfeln; kristallinische Massen wurden niemals beobachtet. Die Würfelflächen sind meist glatt und glänzend, häufig finden sich jedoch auch Anwachsstreifen parallel oder auch diagonal zu den Würfelkanten. Die meisten Würfel haben eine Kantenlänge von $1-2 \mathrm{~mm}$, der gröBte Kristall besab eine solche von $4 \mathrm{~mm}$. 
Der Dysanalyt besitzt makroskopisch eine mäßig gute Spaltbarkeit nach dem „Würfel“, die sich auch im Dünnschliff durch viele Spaltrisse zu erkennen gibt.

Die meisten Kristalle sind stark von Poren durchzogen, die sie im Dünnschliff zuweilen siebartig durchlöchert erscheinen lassen.

Die Würfel sind in frischem Zustand eisenschwarz gefärbt und zeigen einen halbmetallischen Glanz. Bei der Verwitterung werden sie matt und nehmen eine weiblichgraue Farbe an. Sie sind an sich undurchsichtig, werden aber in den allerdünnsten Schliffen durchsichtig. In solchen Schliffen ist die hohe Licht- und geringe Doppelbrechung unschwer zu erkennen. ${ }^{1}$

Der Dysanalyt besitzt das spezifische Gewicht 4.26 (KNop fand 4.13, Hauser 4.21). Seine Härte ist 5.5. Er ist sehr spröde und läBt sich daher leicht pulvern. Er ist unschmelzbar.

Die AufschlieBung geschah zunächst durch Schmelzen mit Kaliumpyrosulfat in folgender Weise:

Im Platintiegel wurden erst etwa $10 \mathrm{~g}$ Kaliumpyrosulfat eingeschmolzen, dann wurde der Dysanalyt zugegeben und mit feingepulvertem Pyrosulfat bedeckt. Durch diese Art der Beschickung erreicht man, daB der Dysanalyt in einer Schmelze nahezu vollständig aufgeschlossen wird, während sich sonst leicht Klumpen bilden, die vom Pyrosulfat nicht angegriffen werden. Das Erhitzen wurde so lange fortgesetzt, bis ein hellgelber, blasenfreier SchmelzfluB entstanden war. Die Schmelze wurde unter Zusatz von Schwefelsäure und Wasserstoffsuperoxyd mit Wasser ausgelaugt, wodurch die Erdsäuren und auch das gebildete Calciumsulfat leicht in Lösung gehen. Der Rückstand wurde abfiltriert, zur Wägung gebracht und die Kieselsäure durch Abrauchen mit FluBsäure bestimmt. Der im Tiegel verbleibende Rückstand wurde wieder mit Pyrosulfat aufgeschlossen und die Lösung der Schmelze mit der anderen vereinigt. Die Lösung wurde doppelt mit kohlensäurefreiem Ammoniak gefällt. Hierbei muB das Wasserstoffsuperoxyd vorher durch Erwärmen zerstört werden, sonst gehen die Erdsäuren teilweise ins Filtrat und fallen beim Eindampfen desselben wieder aus.

Um die Bildung kolloidaler Lösungen zu vermeiden, wurden alle erdsäurehaltigen Niederschläge mit Ammoniumnitrat (2\%) ausgewaschen, was sich besser als das von WeIss und LANDECKER

1 J. Soellner, Die optischen Eigenschaften des Dysanalyts von Vogtsburg und von Schelingen im Kaiserstuhl. Zentralbl. f. Mineral. uswo. 1912, 310. 
empfohlene halbprozentige Ammoniak bewährte. Zur Abscheidung der Erdsäuren wurde die Lösung des Niederschlages in Schwefelsäure vorsichtig mit Ammoniak neutralisiert (es darf hierbei keine Fällung entstehen), dann mit einer reichlichen Menge schwefliger Säure versetzt, mit einem Uhrglase bedeckt und auf dem Wasserbade erwärmt. Titan- und Niobsäure fallen auf diese Weise in leicht filtrierbarer, flockiger Form quantitativ aus. Das Erwärmen wurde so lange fortgesetzt, bis sich die Erdsäuren zu Boden gesetzt hatten.

Wenn die Säure durch Ammoniak nicht genügend abgestumpft wird, bleiben geringe Mengen Titansäure in Lösung, die leicht kolorimetrisch bestimmt werden können; Niob fällt bei genügendem $\mathrm{Zu}$ satz von schwefliger Säure quantitativ aus. Der Niederschlag wurde in verdünnter Schwefelsäure und Wasserstoffsuperoxyd gelöst und die Fällung nochmals wiederholt. Die vereinigten Filtrate wurden eingedampft und mit Ammoniak gefällt. Der Niederschlag wurde in Salpetersäure gelöst und die Lösung zur Trockne verdampft. Aus der stark verdünnten, salzsauren Lösung des Rückstandes wurden die seltenen Erden als Oxalate ausgefällt. Eisen, Mangan, Aluminium und etwa noch vorhandenes Titan wurden zusammen mit Ammoniak gefällt. Das Titan wurde kolorimetrisch bestimmt, das Eisen nach vorausgegangener Reduktion mit Schwefelwasserstoff titriert. Das Mangan wurde durch Kochen mit $10 \%$ iger Ammoniumpersulfatlösung, der etwas Silbernitrat zugesetzt wurde, in Übermangansäure übergeführt und letztere ebenfalls kolorimetrisch bestimmt. Das Aluminium wurde nach verschiedenen Methoden bestimmt. Zuerst wurde nach Entfernung der seltenen Erden nach der Vorschrift von E. T. ALLEN ${ }^{1}$ das Aluminium mit Phenylhydrazin gefällt. Bei anderen Analysen wurde es durch Schmelzen mit Ätznatron vom Eisen getrennt oder auch nach Bestimmung von Eisen und Titan aus der Differenz berechnet.

Das Calcium wurde in der üblichen Weise als Oxalat gefällt und als Oxyd gewogen. Von Magnesium waren nur Spuren vorhanden. Die Alkalibestimmung wurde nach AufschlieBung mit FluBsäure vorgenommen, da sich der Dysanalyt nach der Methode von L. Sмrтн nicht aufschlieBen läßt.

Kleinere Mengen Dysanalyt lassen sich auch mit konzentrierter

1 E. T. Allen, Precipitation and Separation by weak organic Bases. Journ. Amer. Chem. Soc. 25 (1903), 421. 
Schwefelsäure aufschließen. ${ }^{1}$ Etwa 0.5 g Substanz wurden in einer mit einem Ubrglas bedeckten Platinschale mit $15 \mathrm{ccm}$ konzentrierter Schwefelsäure erhitzt, bis diese zu stoBen anfing, was nach etwa 20 Minuten eintrat. Die Schale wurde in einem großen Becherglas mit etwa $600 \mathrm{ccm}$ Wasser übergossen und 24 Stunden stehen gelassen. Nach dieser Zeit war fast alles in Lösung gegangen. Es wurde nun Wasserstoffsuperoxyd zugesetzt und in derselben Weise wie vorher verfahren.

Die Trennung der Niob- und Titansäure haben wir nach der Methode von Weiss und LandeckeR ${ }^{2}$ durch Schmelzen mit Soda und Salpeter ausgeführt, da diese sich nach vielfachen Vorversuchen als die beste erwiesen hatte. $\mathrm{Ob}$ der Zusatz von Salpeter einen wesentlichen Einfluß auf die Trennung hat, erscheint uns fraglich, doch haben wir keine Versuche darüber angestellt. Nach HAUSER ${ }^{3}$, sowie nach RUFF und SchmLER ${ }^{4}$ ist er gänzlich belanglos.

Gute Ergebnisse zeigte auch die von DemarçaY ${ }^{5}$ herrührende Methode in folgender Ausführung. Die Erdsäuren werden unter Umrühren und gelindem Erwärmen (Mikrobrenner) in Flubsäure gelöst, mit etwas Wasser verdünnt, mit Pḥenolphthalein versetzt und zum Sieden erhitzt. In die kochende Lösung läBt man aus einer Bürette Ammoniak (etwa 8-9\% ig) zufließen, bis die Lösung deutlich rot gefärbt ist. Der zuerst entstehende Niederschlag löst sich beim Kochen größtenteils oder auch vollständig wieder auf. Beim Erkalten scheidet sich die Niobsäure als dicker, flockiger Niederschlag ab. Dieser wurde abfiltriert (Glas wird hierbei nicht angegriffen) und mit heißer Ammonnitratlösung ausgewaschen. Das Filtrat wird in einer Platinschale aufgefangen, einige Tropfen FluBsäure zugesetzt und das Verfahren wiederholt. Die das Titan enthaltenden Filtrate werden mit einem reichlichen Überschuß von

1 F. W. Mar, On the so-called Perofskite from Magnet-Cove (Arkansas). Amer. Journ. Sei. [3] 40 (1890), 403.

${ }^{2}$ L. Weiss und M. Landecker, Untersuchungen über die quantitative Bestimmung der Erdsäuren. Z. anorg. Chem. 61 (1909), 65.

${ }^{3}$ O. HAUSER, Über die Hydrosole der Niobsäure und der Tantalsäure und die Trennung des Niobs und Tantals nach Weiss-Iandecker. Zeitschr. angew. Chem. 1912, 100.

4 O. Rupr und E. Schincrr, Über Tantal- und Niobpentafluorid, sowie über die Reindarstellung der Tantal- und Niobsäure. Z. anorg. Chem. 72 (1911), 353.

5 E. Demarçay, Sur la séparation du titane d'avec le niobium et le zirconium. Compt. rend. 100 (1885), $\mathbf{7 4 0 .}$ 
Ammoniak und mit festem Ammonnitrat versetzt und längere Zeit gekocht, wodurch das Titan ausgefällt wird. Durch weiteres Kochen prüft man, ob im Filtrat noch Titan vorhanden ist. Die Reaktion mit Wasserstoffsuperoxyd ist in diesem Fall wegen der Anwesenheit von FluBsäure-nicht anwendbar. Die Niederschläge können ohne weiteres geglüht werden. Auf diese Weise erreicht man, daB das Niob quantitativ, wenn auch titanhaltig abgeschieden wird; doch läBt sich der Titangehalt leicht kolorimetrisch bestimmen.

Als Mittel mehrerer Analysen erhielten wir für die Zusammensetzung dieser Dysanalytprobe die unter 1 angeführten Zahlen.

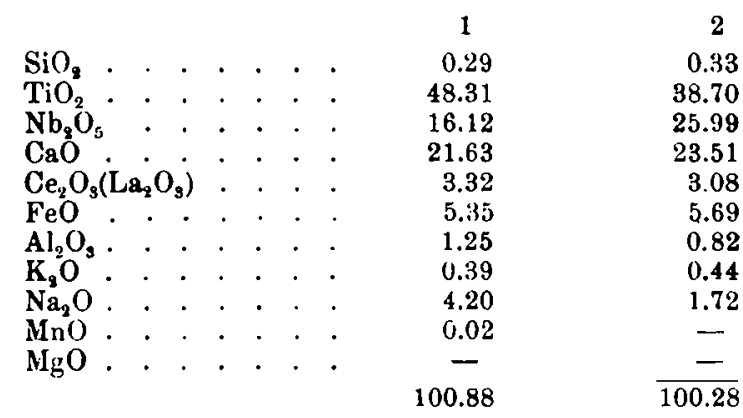

Eine nach Demarçay ausgeführte Trennung der Erdsäuren ergab $47.37 \% \mathrm{TiO}_{2}$ und $16.71 \% \mathrm{Nb}_{2} \mathrm{O}_{5}$, zusammen $64.08 \%$ Erdsäuren.

Zur Kontrolle wurde die Titansäure noch kolorimetrisch bestimmt.

Es wurde etwa $0.1 \mathrm{~g}$ Dysanalyt mit Kaliumpyrosulfat aufgeschlossen, die Lösung der Schmelze mit Schwefelsäure und Wasserstoffsuperoxyd auf $500 \mathrm{ccm}$ aufgefüllt und im Hillebrandschen Kolorimeter mit einer Titanlösung, die im Kubikzentimenter $0.5 \mathrm{mg}$ $\mathrm{TiO}_{2}$ enthielt, verglichen. Als Mittelwert wurden $48.0 \% \quad \mathrm{TiO}_{2}$ gefunden.

Da eine kolorimetrische Titanbestimmung in einer selbstgesammelten Probe von den früher gefundenen Werten stark abwich, wurde auch von dieser Probe eine vollständige Analyse ausgeführt. Die Erdsäuren wurden hierbei nach der Demazçayschen Methode getrennt; das Titan wurde auBerdem in besonderen Proben mit dem AUTENRIETH-KöNIGSBERGER schen Kolorimeter bestimmt. Unseren Erfahrungen nach ist es überhaupt am richtigsten, beide Säuren zusammen zu bestimmen und den Titangehalt in einer besonderen Probe kolorimetrisch zu ermitteln. Man schlieBt dadurch alle mehr oder weniger fraglichen Trennungsmethoden aus. 
Die hierbei erhaltenen Zablen sind unter 2 angegeben. Die direkte kolorimetrische Bestimmung der Titansäure ergab für diese Probe als Mittel $37.4 \% \mathrm{TiO}_{2}$.

Um zu entscheiden, ob die Verschiedenheit in der Zusammensetzung oder an der Art der Vorbehandlung liegt, wurde frischer, fein zerriebener Dysanalyt einige Stunden mit konzentrierter Salzsäure gekocht und die Lösung analysiert.

In Lösung gegangen waren $7,32 \%$, davon waren

$$
\begin{aligned}
& \mathrm{TiO}_{8} \text {. . . . . } \quad 1.69 \\
& \mathrm{Nb}_{2} \mathrm{O}_{3} \cdot+. \quad \cdot \quad 1.52 \\
& \mathrm{FeO} \text {. . . . . } 2.26 \\
& \mathrm{CaO} \cdot \cdot \cdot \cdot \frac{1.59}{7.32}
\end{aligned}
$$

Aus diesen Zahlen ergibt sich ohne weiteres, daB die Unterschiede in der Zusammensetzung der beiden Proben ursprüngliche sind und nicht etwa erst durch eine verschiedene Behandlung mit Säure hervorgerufen wurden. Im letzteren Falle hätte der Gehalt an Kalk und Eisenoxydul viel stärker abnehmen müssen als der Gehalt an Niobsäure. Wir müssen also annehmen, daB die $\mathrm{Zu}$ sammensetzung der einzelnen Dysanalytkristalle an verschiedenen Stellen des Vorkommens verschieden ist.

Wenn der Niobgehalt des Dysanalyts so stark wechselt, so ist es an sich natürlich nicht unmöglich, $\mathrm{daB}$ er auch einmal so gering wird, wie ihn HAUSER bestimmt hat. Im allgemeinen ist er jedoch so erheblich, dab es durchaus gerechtfertigt ist, den Dysanalyt als ein selbständiges Mineral anzuselıen und ihm demgemäB auch seinen alten Namen zu belassen.

Freiburg i. Br., Chemisches Universitätslaboratorium (Abllg. d. naturu.math. Fak.).

Bei der Redaktion eingegangen am 20. Mai 1913. 